dry, and the colorimeter must be held far enough from the reflecting surface to insure even illumination. Naturally, the test tubes must be clean, but there is far less trouble from dirty glass in this instrument than in other colorimeters.

The instrument offers certain advantages over other colorimeters. To mention only a few of these, it costs only about a dollar to make; it is possible to read accurately much paler colors than with the Duboscq or Hellige colorimeters; and, as no cement is used in its construction, strongly acid or alkaline solutions can do the instrument no harm. Another

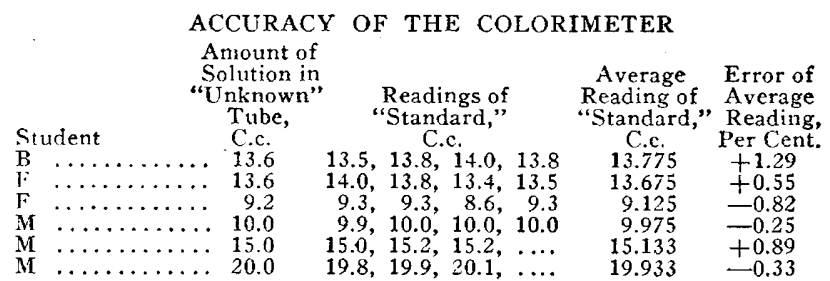

point of interest worthy of note is the fact that with this instrument subjective error is reduced to a minimum, because the scale to be read is hidden completely during the matching of the colors.

We have used the instrument repeatedly and with perfect satisfaction for the determination of phenolsulphonephthalein, ammonia, urea, creatinin, and total nitrogen in urine; urea, nonprotein nitrogen, uric acid, creatinin and creatin, and sugar in blood; and protein and lactose in milk.

DEATH FROM PRESSURE ON LARYNX BY EXTREME ANTERIOR CURVATURE OF CERVICAL SPINE

\footnotetext{
Alfred H. Noehren, M.D., Buffalo

Fellow of the American College of Surgeons
}

Miss U., aged 66, was admitted to the Lutheran Church Home as an inmate about twenty years ago. Since that time, she had been in fairly good health, except that she had experienced constant difficulty in swallowing. Thus she had been unable to eat her oatmeal without picking out any lumps that might be in it. She had also been markedly hunchbacked ever since she had been in the institution. The cause of this deformity could not be ascertained.

I saw her for the first time, the morning of Jan. 5, 1918. During the two previous days, her difficulty in swallowing had markedly increased and for twelve hours she had been unable to swallow anything.

Only a very superficial examination was possible, as the patient was in great distress and could not breathe except when curled up on her left side. The most obvious symptom was her extreme dyspnea, not a rapid breathing as in pneumonia, but a labored and rather slow breathing, as though there were some obstruction in the respiratory tract. Examination of heart and lungs showed them to be normal, but very little air was entering the lungs. On looking into her mouth, I saw a bulging on the posterior pharyngeal wall, which felt hard to the palpating finger.

Further examination was made impossible by her condition but a diagnosis of pressure on some part of the respiratory tract was made. Death occurred about six hours later.

Postmortem examination, made by Dr. Mueller, pathologist of the Deaconess Hospital, revealed the cause of the dyspnea and dysphagia. The marked kyphosis in the dorsal region caused a compensatory lordosis in the lumbar and cervical regions, the most anterior portion of the latter consisting of the third and fourth cervical vertebrae. This anterior curvature came forward directly above the larynx and the opening of the esophagus, and pressed the epiglottis down on both of them, thus practically shutting them off. It occupied so much of the usual pharyngeal space that when the finger was introduced into the mouth, it felt like a large osteoma filling the mouth and pharynx. A thorough examination of the interior of the chest revealed nothing abnormal except a reddening of the mucous membrane of the trachea, with a moderate amount of semipurulent exudate.

The sequence of events which led to the patient's death was probably somewhat as follows: With the settling of the vertebrae, which occurs in old age, the abnormal curves in the spine were exaggerated, until the cervical convexity came forward to such an extent that it almost shut off the glottis and the esophagus, causing an edema of the larynx that quickly caused her death.

If an exact diagnosis had been possible before death, a tracheotomy might have been indicated, but probably would not have saved the patient's life on account of her age and poor condition when first seen.

1196 Main Street.

\section{IMPROVED MOUTH-GAG AND TONGUE-DEPRESSOR}

$$
\text { J. J. Moyer, M.D., Oakland, Calif. }
$$

This invention relates to improvements in mouth specula and more particularly to an improved tongue-depressor. Its object is to provide a tongue-depressor which is instantaneously removable from the mouth-gag. This tongue-depressor may be adjusted for depth to suit the mouth, and has an oscillatory mounting, leaving the operator free to move the tongue-depressor from side to side without affecting the adjustment of the gag or removing the depressor from its holding means,

The particular advantage of the device is the ease with which the tongue-depressor is manipulated, in and out or from side to side in an instant.
The ratchet on the gag affords adjustment of the mouth to any desired opening. By the pressing of a button on the side, the ratchet is released.

In the hands of my associates and myself, this instrument gives great satisfaction.

Fourteenth and Jefferson Streets.

First American Book on Military Medicine.-John Jones (1729-1791), who had been a surgeon. of French troops in the war of 1758 and afterwards rendered worthy service in the Revolution, published a treatise on wounds and fractures in 1775 , which was the first surgical work published in this country, and, through its appendix on camp and military hospitals, the first American book on military medicine.Lieut.-Col. C. C. McCulloch, Jr., U. S. Arny: The Scientific and Administrative Achievement of the Medical Corps of the United States Army, the Scientific Monthly. 